\title{
Quadrature Subharmonic Coupled Oscillators for a 60GHz SiGe Scalable Phased Array
}

\author{
James F. Buckwalter, Aydin Babakhani, Abbas Komijani, and Ali Hajimiri \\ California Institute of Technology, Pasadena, CA 91125
}

\begin{abstract}
This paper describes an integrated coupled oscillator array in SiGe for millimeter wave applications. The design focuses on scalable radio architectures where multiple dies are tiled to form larger arrays. A $2 \times 2$ oscillator array for a $60 \mathrm{GHz}$ transmitter is designed with integrated power amplifiers and antennas. The $2 \times 2$ array demonstrates a $200 \mathrm{MHz}$ locking range and $1 \times 4$ array formed by two adjacent chips has a $60 \mathrm{MHz}$ locking range. The phase noise of the array is below $-110 \mathrm{dBc} / \mathrm{Hz}$ at a $1 \mathrm{MHz}$ offset.
\end{abstract}

Index Terms - Phased Array, Coupled Oscillator, Millimeter Wave, Silicon Germanium.

\section{INTRODUCTION}

Unlicensed operation at the $59-65 \mathrm{GHz}$ band is stimulating unique radio architectures for millimeter wave integrated circuits. Transceiver circuits that operate at millimeter wave frequencies were demonstrated with $\mathrm{SiGe}$ [1][2]. However, the integration of a complete phased array transceiver with antennas on a single chip offers new design choices and opportunities. This paper demonstrates a coupled oscillator array for millimeter wave silicon integrated circuits.

Coupled oscillators are suited for fully integrated phased array circuits. First, the small wavelength at $60 \mathrm{GHz}$ allows fully integrated arrays on a single die. Scalable architectures are particularly appropriate for creating larger arrays by tiling several dies. In Fig. 1, scalability is demonstrated for a phased array transmitter. The phased array requires phase coherence between all elements. Since each chip requires frequency generation, neighboring chips are locked to ensure phase coherence. If oscillators are located at each antenna element, injection locking can lock the oscillators both on-chip and between chips.

Furthermore, injection locked coupled oscillators offer a new approach to the distribution of the carrier frequency between different elements. Previous integrated phased arrays provide sixteen phases across the entire chip, resulting in an excessive amount of wiring and power consumption [3]. Global frequency distribution becomes increasingly difficult at millimeter frequencies.

In this transmitter implementation, a $2 \times 2$ array of transmitter cells is integrated on a single chip with antennas.

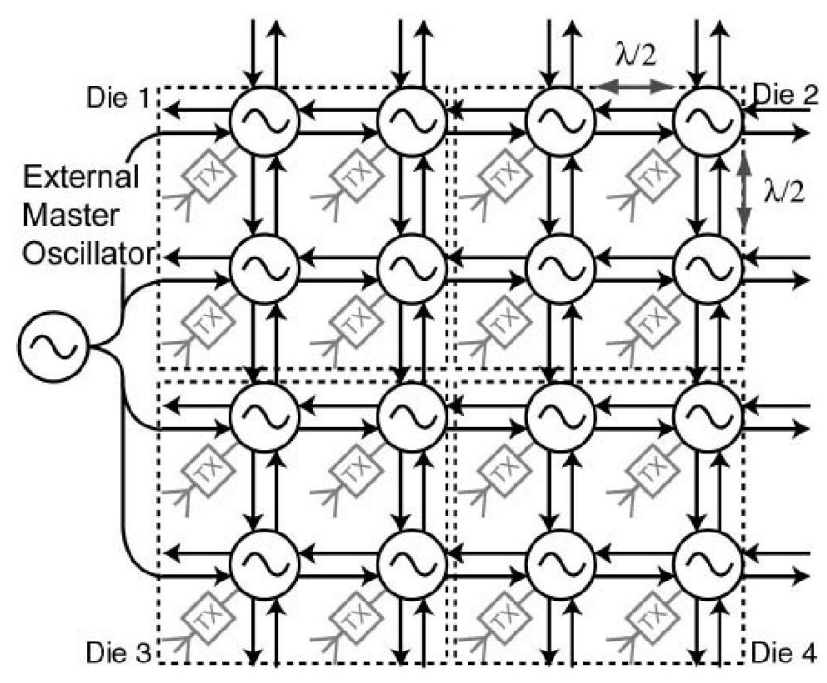

Fig. 1. Coupled oscillator approach for fully integrated phasedarray transmitter. Each oscillator drives a transmit chain with a DAC controlled phase shifter, power amplifier and antenna.

The design of the coupled oscillator network is motivated by demonstrating array scalability as well as avoiding the on-chip coupling that occurs with power-amplifiers and antennas. The measured results show that the phase noise of each element can be suppressed when locked to an external array across a single chip as well as across two chips.

\section{COUPLED Oscillators}

Adler described the unilateral locking phenomena in coupled oscillators [4] and Kurokawa later studied the microwave dynamics [5]. The phase relationship between neighboring oscillators is

$$
\Delta \theta=\sin ^{-1}\left(\frac{2 Q}{\omega_{o}} \frac{I_{o s c}}{I_{i n j}} \Delta \omega\right) .
$$

where $\Delta \theta$ and $\Delta \omega$ are the phase difference and frequency detuning between the two oscillators, $\omega_{\mathrm{O}}$ is the carrier frequency, $Q$ is the quality factor for the oscillator, and $I_{\text {osc }}$ 


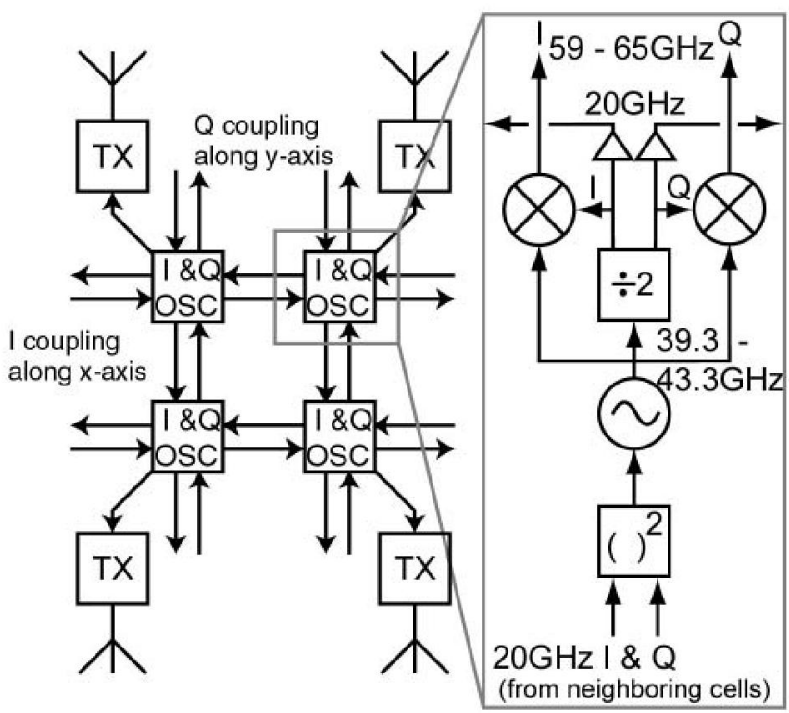

Fig. 2. Implemented coupled oscillator topology. The oscillators are coupled along two dimensions. The coupling network operates at one-third the carrier frequency.

and $I_{i n j}$ is the oscillator and injection current. The locking range is defined as twice $\Delta \omega_{m}=\omega_{\mathrm{o}} I_{\text {inj }} / 2 Q I_{\text {osc }}$ and allows the phase difference to vary from -90 to 90 degrees. Bilateral oscillator arrays was first proposed by Stephan [6]. Later, York et al. proposed coupled oscillators for electronic beam steering through injection at the periphery of the array [7]-[8]. The effect of bilateral injection locking is to modify the behavior in (1) as described in [8].

The on-chip process and voltage variations between different oscillators introduces phase errors between neighboring elements. Instead of exploiting the phase relationship in (1) to control the beam angle, this work instead relies on injection locking primarily as a means to distribute and phase lock the carrier signal across a single chip or between neighboring chips. The locking range consequently must be large enough to satisfy the device process variations in a particular technology. Additionally, slight supply variations shift the natural frequencies of neighboring oscillators. Additionally, the digitally controlled phase shifters are important for modulation of the array.

\section{SCALABLE 2D COUPLED OSCILlatoR}

The basic coupled oscillator topology is shown in Fig. 2. The $2 \times 2$ phased array contains four oscillators at each transmit (TX) stage. The block diagram demonstrates the implementation of the oscillator cell. Each oscillator is designed to operate at $40 \mathrm{GHz}$. Isolating the oscillator from the power oscillator and antenna is critical because on-chip coupling is difficult to accurately model and can greatly impact performance. By shifting the oscillator frequency to $40 \mathrm{GHz}$, the unwanted coupling is reduced. The oscillator is mixed with a divided carrier frequency at $20 \mathrm{GHz}$ to achieve the $60 \mathrm{GHz}$ in-phase (I) and quadrature (Q) signals.

Coupling between chips requires considering an interconnect. If wirebonds are used to provide the interconnect, frequencies above $20 \mathrm{GHz}$ incur high losses. Consequently, we choose to injection lock at a subharmonic. We propose an I/Q scheme for coupling within a 2-D array of oscillators. In Fig. 2, the $20 \mathrm{GHz}$ I signal is coupled in the EastWest direction while the Q signal is coupled along the North-South direction. Since the injected signal is a subharmonic of the oscillator, the injected frequency is doubled at the oscillator. Injection locking with quadrature subharmonic signals increases the 2nd harmonic power since $[\sin (\omega t)+\cos (\omega t)]^{2}=\sin (2 \omega t)$.

Transmission lines form the subharmonic interconnects between the neighboring oscillator cells. Because the large metal grounds of the transmission lines can disturb the radiated field from the on-chip antennas, the ground plane is periodically severed. The antenna radiation is also strongly affected by the presence of power supply lines to each cell. Consequently, the power lines were all designed to run perpendicular to the antenna plane.

A circuit schematic for the frequency doubler and oscillator is shown in Fig. 3. The I/Q signals are picked up by a receiver that drives a pair of bipolar devices. These bipolar transistors are biased to generate strong second harmonic content through rectification. Changing the bias allows control over the injected current at the second harmonic.

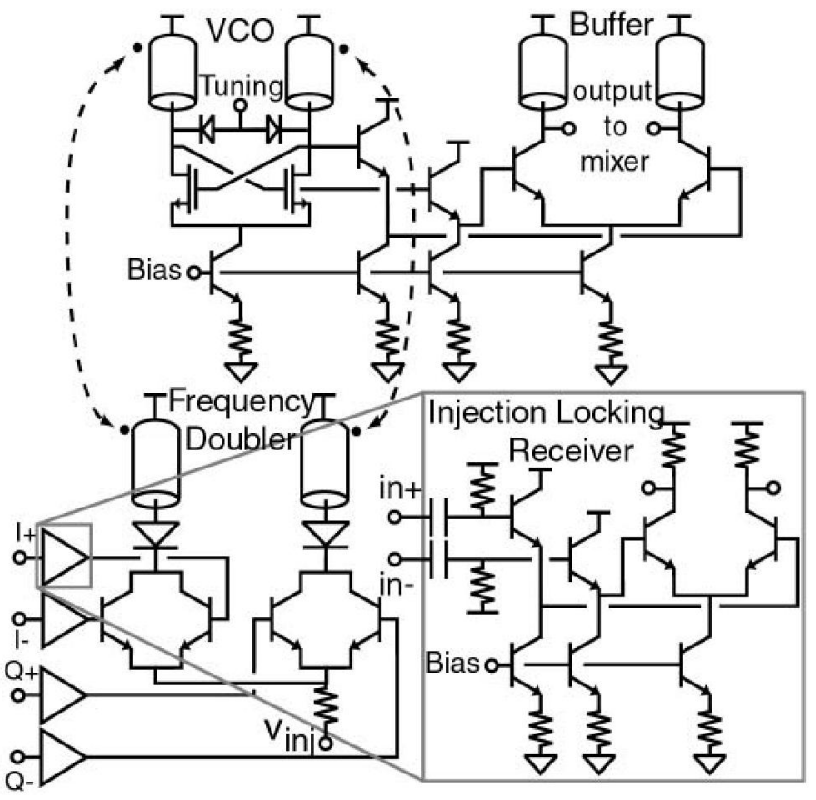

Fig. 3. Current injected into the VCO at subharmonic and oscillator frequency. 


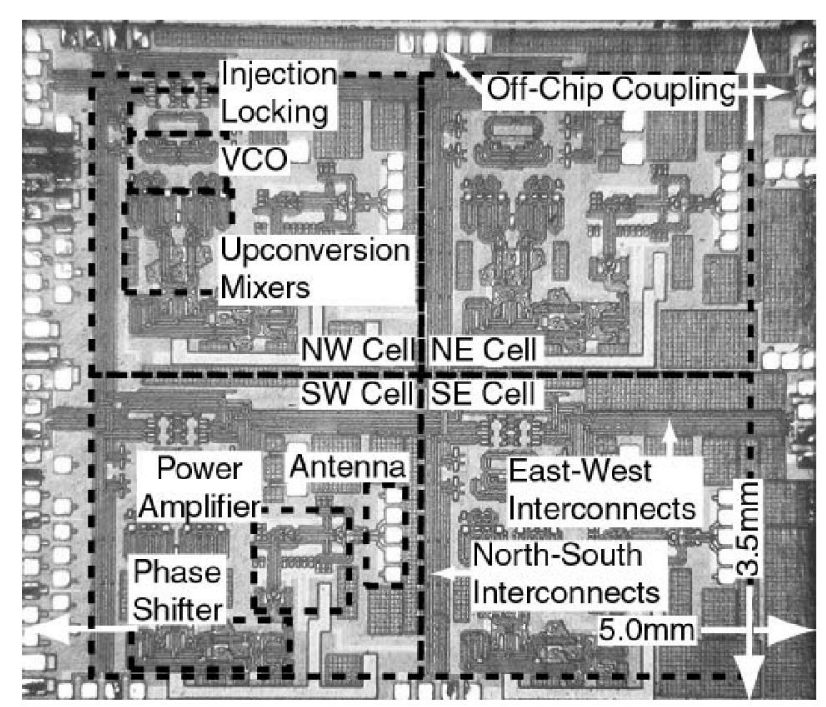

Fig. 4. Chip microphotograph for complete $60 \mathrm{GHz}$ transmitter with coupled oscillator array.

The frequency doubler output is coupled into the VCO core with a coupled transmission line. The coupled transmission line is part of the VCO tank circuit and has less parasitic loading but limits the coupling strength. In this case, the geometry of the transmission line tank of the VCO limited the coupled transmission line section to $100 \mu \mathrm{m}$. The coupling at $40 \mathrm{GHz}$ is $-10 \mathrm{~dB}$ for this length. Consequently, a large amount of injection current is lost through the coupled transmission line. Interestingly, the coupling at $20 \mathrm{GHz}$ is $-15 \mathrm{~dB}$, providing rejection of the subharmonic frequency. The tuning range of the VCO is over $3 \mathrm{GHz}$, about $8 \%$ of the carrier frequency. Notably, the oscillator self-mixing enhances the tuning range at $60 \mathrm{GHz}$.

\section{RESULTS}

The coupled oscillator array was constructed in IBM $8 \mathrm{HP}$, a $0.12 \mu \mathrm{m}$ SiGe process with bipolar and CMOS devices. The maximum $f_{T}$ of the bipolar devices is $210 \mathrm{GHz}$. The array is shown in Fig. 4 and occupies an area of $3.5 \mathrm{~mm}$ by $5 \mathrm{~mm}$. The chip also includes a DAC controlled phase shifter, power amplifier, and antenna located in each cell.

The VCO frequency varies between 36.5 and $39.5 \mathrm{GHz}$, falling $2.5 \mathrm{GHz}$ below the desired range of 39 to $42.5 \mathrm{GHz}$. The injection locking characteristics are measured as a function of injection power. In Fig. 5, the carrier frequency is measured as a function of the injected subharmonic fre-

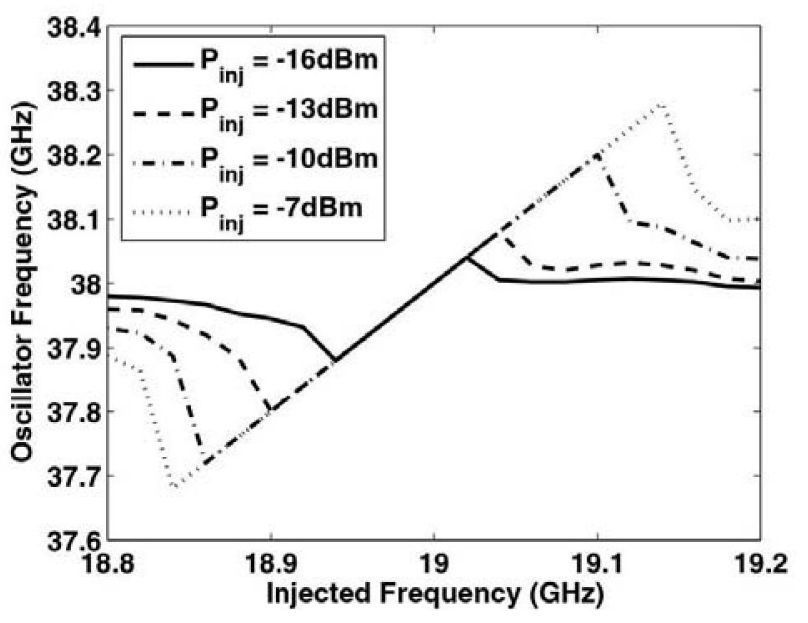

Fig. 5. Locking range for the VCO as a function of injected power.

quency. At $P_{i n j}=-16 \mathrm{dBm}$, the locking range is around $60 \mathrm{MHz}$ and increases to $320 \mathrm{MHz}$ at $P_{i n j}=-7 \mathrm{dBm}$.

In Fig. 6 , the phase noise of the $2 \times 2$ coupled oscillator array structure is shown without injection locking. In this case, the average phase noise of each oscillator is around -

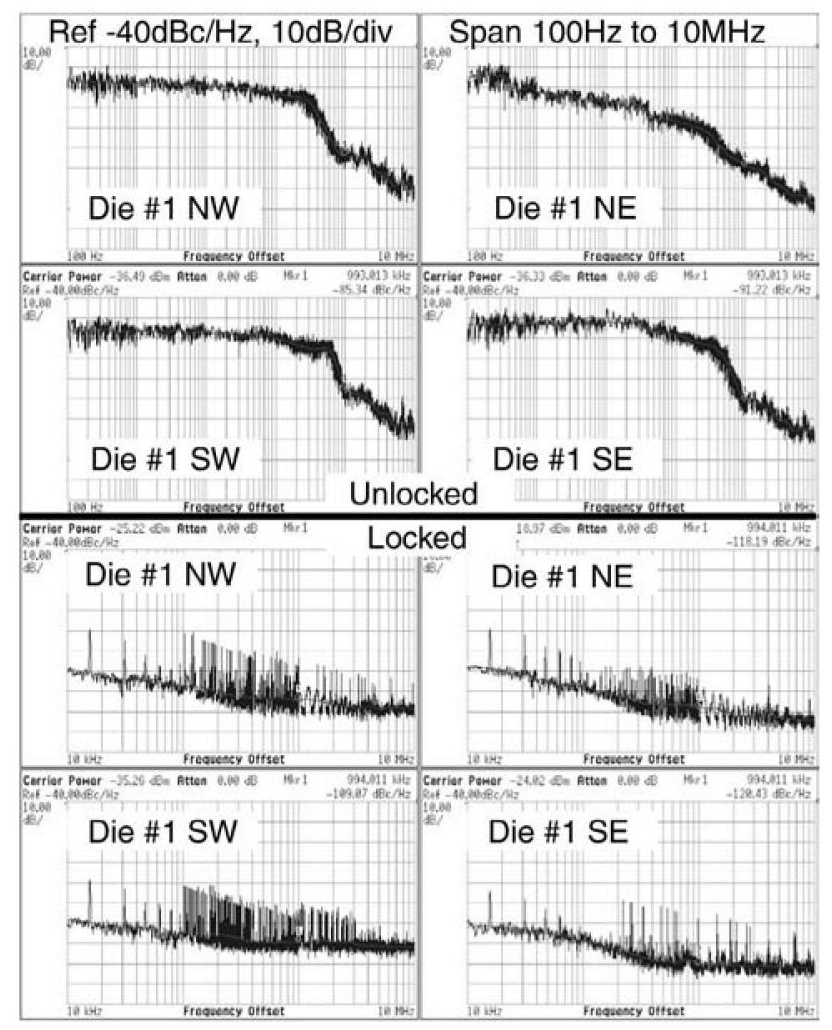

Fig. 6. Phase noise of each oscillator in $2 \times 2$ single chip array without (Top) and with (Bottom) injection locking. 
$93 \mathrm{dBc} / \mathrm{Hz}$ at $1 \mathrm{MHz}$ offset. Next, a $-10 \mathrm{dBm}$ external reference is injected at the NW and SW oscillators. The oscillator phase noise was consecutively measured without changing the operating conditions. The phase noise of the injection locked VCOs is around $-114 \mathrm{dBc} / \mathrm{Hz}$ at $1 \mathrm{MHz}$ offset and the phase noise of the reference oscillator. The locking range for the array under these conditions is about $200 \mathrm{MHz}$. The operation of the locked array is limited by the natural frequency and power variations for each oscillator due to process and circuit mismatches. This affects the phase noise of each oscillator in the array.

The normalized phases of each oscillator can also be measured at $20 \mathrm{GHz}$ with a high-speed sampling scope. The externally injected signal is used to trigger the highspeed scope. The phase progression of each oscillator is demonstrated in Fig. 7. Each oscillator demonstrates a phase variation of roughly -60 to 70 degrees over the $220 \mathrm{MHz}$ locking range. This locking range is measured at the subharmonic frequency but the phase variation is calculated for the actual carrier frequency. Voltage variations strongly influence the locking range. The southern oscillators demonstrate a smaller locking range.

Finally, a $1 \times 4$ oscillator array is measured in Fig. 8 with an external injection signal of $-10 \mathrm{dBm}$ at the NW oscillator of die \#1. The locking range under these conditions is roughly $60 \mathrm{MHz}$ and is reduced because only the in-phase signals are used to injection lock the array. The low phase noise across the two chips is encouraging for demonstrating largere arrays by locking on-chip oscillators to off-chip reference signals.

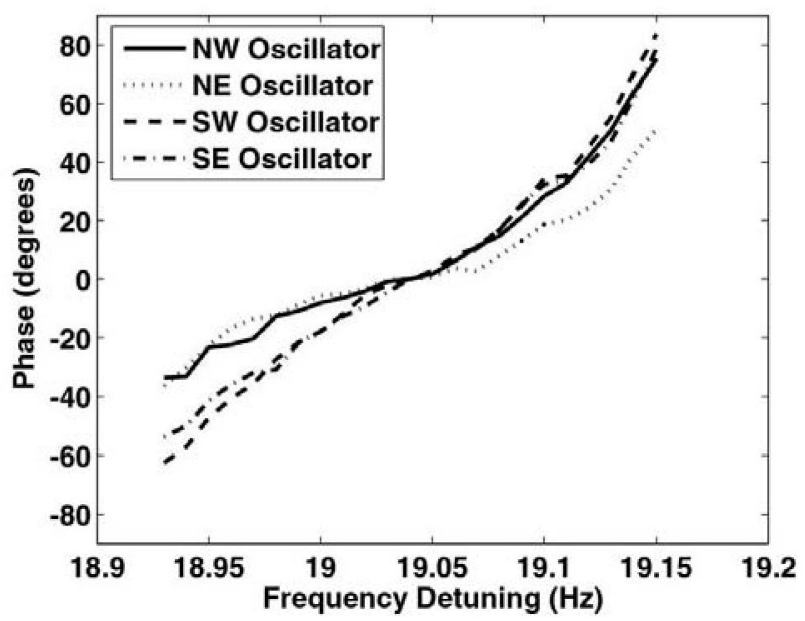

Fig. 7. Phase of each oscillator in array across locking range.

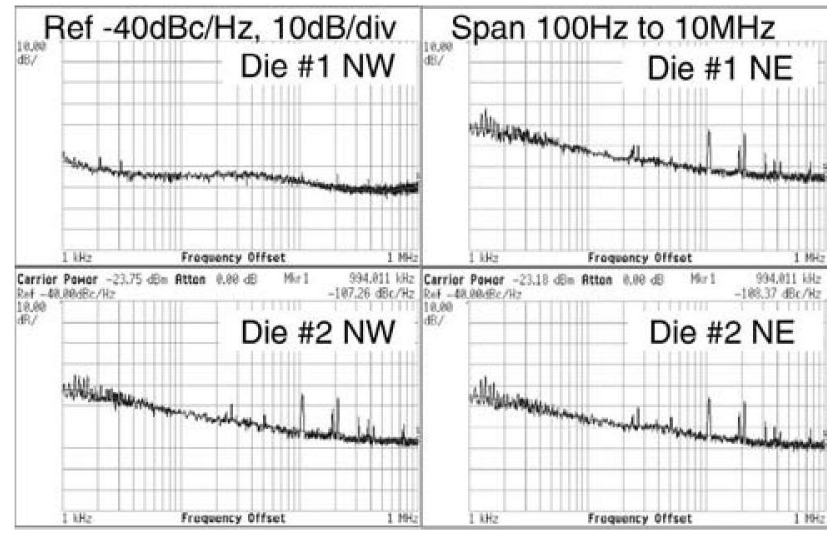

Fig. 8. Phase noise of oscillators across a $1 \times 4$ array with 2 chips.

\section{CONCLUSION}

This paper describes the implementation of a coupled oscillator array for submillimeter applications. The coupled oscillator employs quadrature subharmonic injection locking to couple neighboring chips connected through wirebonds. Measurement of the oscillator array demonstrate reduced phase noise when locked to an external reference. The locking range was measured to be $220 \mathrm{MHz}$ for the oscillators on a single die and is limited by the process and voltage variations that exist over the large die area.

\section{REFERENCES}

[1] S. Reynolds, et al., "60GHz Transceiver Circuits in SiGe Bipolar Technology," IEEE Int. Conf. on Solid-State Circuits, pp. 442-444, Feb. 2004.

[2] A. Babakhani, et al., " $77 \mathrm{GHz}$ Four Element Phased Array Receiver with On-Chip Dipole Antennas in Silicon," to appear at IEEE Int. Conf. on Solid-State Circuits, Feb. 2006.

[3] H. Hashemi et al., "A 24-GHz SiGe Phased Array ReceiverLO Phase Shifting Approach," IEEE T. of Microwave Theory and Techniques, vol. 53, pp. 614-626, Feb. 2005.

[4] R. Adler, "A Study of Locking Behavior in Oscillators" Proc. of the IEEE, vol. 61, pp. 1380-1385, Oct. 1973.

[5] K. Kurokawa, "Injection Locking of Microwave Solid-State Oscillators" Proc. of the IEEE, vol. 61, pp. 1386-1410, Oct. 1973.

[6] K. D. Stephan, "Inter-Injection-Locked Oscillators for Power Combining and Phased Arrays." IEEE T. of Microwave Theory and Techniques, vol. 34, pp. 1017-1025, Oct. 1986.

[7] R. A. York and R. C. Compton, "Measurement and Modelling of Radiative Coupling in Oscillator Arrays," IEEE T. of Microwave Theory and Techniques, vol. 34, pp. 438-444, March 1989.

[8] R. A. York and T. Itoh, "Injection and Phase-Locking Techniques for Beam Control," IEEE T. of Microwave Theory and Techniques, vol. 46, pp. 1920-1929, Nov. 1998. 